\title{
Temporal Precision of Spike Trains in Extrastriate Cortex of the Behaving Macaque Monkey
}

\author{
Wyeth Bair* \\ Christof Koch \\ Computation and Neural Systems Program, California Institute of Technology, \\ Pasadena, CA 91125 USA
}

How reliably do action potentials in cortical neurons encode information about a visual stimulus? Most physiological studies do not weigh the occurrences of particular action potentials as significant but treat them only as reflections of average neuronal excitation. We report that single neurons recorded in a previous study by Newsome $e t$ al. (1989; see also Britten et al. 1992) from cortical area MT in the behaving monkey respond to dynamic and unpredictable motion stimuli with a markedly reproducible temporal modulation that is precise to a few milliseconds. This temporal modulation is stimulus dependent, being present for highly dynamic random motion but absent when the stimulus translates rigidly.

\section{Introduction}

Because the mean firing frequency of a neuron, typically averaged over a fraction of a second or more, in response to a visual stimulus is reproducible under identical stimulus conditions and varies predictably with stimulus parameters, it is widely held to be the primary variable relating neuronal response to visual experience (Adrian 1928; Lettvin et al. 1959; Werner and Mountcastle 1963; Barlow 1972; Henry et al. 1973). Accordingly, many studies hold a stimulus parameter constant during an experiment, measure large variations in firing frequency across different trials and high within-trial variation in interspike intervals, and conclude that the microstructure of spike trains is essentially random (Schiller $e t$ al. 1976; Heggelund and Albus 1978; Tolhurst et al. 1981, 1983; Vogels et al. 1989; Softky and Koch 1993; Shadlen and Newsome 1994). A few studies have emphasized that cells in mammalian visual cortex responding to moving patterns show stimulus-locked temporal modulation, sometimes referred to as "grain" response (Tomko and Crapper 1974; Hammond and MacKay 1977; Gulyas et al. 1987; Snowden et al. 1992). However,

\footnotetext{
${ }^{*}$ To whom all correspondence should be addressed, at: Howard Hughes Medical Institute and Center for Neural Science, New York University, 4 Washington Place, Rm. 809, New York, NY 10003.
} 
the time scale and stimulus dependency of this type of modulation have not been characterized at the trial-to-trial level. Stimulus-locked modulation has also been shown to exist in visual cortex for static patterns (Richmond et al. 1987, Richmond et al. 1990). Studies that have explicitly addressed the temporal frequency profiles of LGN and visual cortical neurons (Derrington and Lennie 1984; Foster et al. 1985; Lee et al. 1989; Levitt et al. 1994) have typically used drifting sinusoidal gratings-stimuli that rarely induce temporal modulation in the output of MT cells (Sclar et al. 1990; J. Anthony Movshon, personal communication).

We analyzed data from an earlier series of experiments by Newsome et al. (1989; see also Britten of al. 1992) that linked the response of wellisolated single units in extrastriate area MT to the psychophysical performance of macaque monkeys. We show that the dynamic random dot stimuli employed in these studies, largely composed of spatially and temporally broad-band noise (Britten et al. 1993), can produce highly modulated responses in MT neurons, and we present a brief characterization of the neuronal response in terms of temporal precision, reliability, and power spectra.

The present study makes use of data that were collected many years ago, and the analysis is limited in some ways because the randomization seeds for the stimuli (see Methods) were not stored. Random dot stimuli have been used for the study of motion perception since the early 1970s, and yet even recent electrophysiological studies of the response of MT neurons to random dot stimuli (Snowden et al. 1992; Britten et al. 1993) have not found or not emphasized the temporal aspects of the neuronal response. We therefore think it is useful to point out the temporal properties of the responses to a random dot stimulus as an aid for the development of further experiments that could make use of reverse correlation or stimulus reconstruction techniques (McLean and Palmer 1989; Bialek et al. 1991).

\section{Methods}

Details of the stimulus generation and experimental paradigm are summarized here. For a full account of the electrophysiological methods, see Britten et al. (1992).

2.1 Experimental Procedures. The dynamic dot stimulus consisted of $0.1^{\circ}$ diameter dots plotted asynchronously for $2 \mathrm{sec}$ at a density of 16.7 dots/degree ${ }^{2} / \mathrm{sec}$ on a large-screen CRT monitor (Hewlett-Packard $1321 \mathrm{~B}$ or XYtron A21-63; P4 phosphor, $0.2 \mathrm{~cd} / \mathrm{m}^{2}$ mean luminance). The dots were illuminated for $150 \mu \mathrm{sec}$. The diameter of the circular aperture (between $5^{\circ}$ and $15^{\circ}$ ) in which the dots appeared was optimized for the receptive field of the neuron, so neurons having smaller receptive fields were stimulated with fewer total dots. A coherent motion signal was 
introduced in the display by altering the probability $c$ that a given dot would be displayed with a particular spatial and temporal offset relative to a previous dot. The temporal offset was $45 \mathrm{msec}$, and the spatial offset was adjusted to match the velocity preference of the neuron (or to oppose it by $180^{\circ}$ for null direction trials). The probability $c$ is referred to as the "motion coherence level." The probability that a sequence of $n$ dots would be generated that carried the motion signal was $c(n-1)$. The coherence is varied from 0 to 1 . At $c=0$, all dots are plotted randomly, while at $c=1$ the display appears to be a rigid sparse dot pattern that translates at the neuron's preferred speed and direction.

The exact pattern of dots in a particular stimulus was determined by the seed value used to initialize the random number generator. The seed was occasionally set to a predetermined constant so that many responses to the same dot pattern could be recorded, but identical stimuli were always interleaved with stimuli having other seeds, at other coherence levels, or moving in the other direction. All trials having a constant seed were grouped together and analyzed, but the values of the constant seeds were not stored, precluding stimulus reconstruction or the use of reverse correlation methods.

During the $2 \mathrm{sec}$ stimulus presentation, occurrence times of action potentials were recorded with a $1 \mathrm{msec}$ resolution. In the worst case, this discretization would add $0.5 \mathrm{msec}$ to our standard deviation measure of temporal jitter (see Section 3.1 below). Other sources of error in the recorded time of action potentials relative to stimulus onset are small compared to the quantization error. The monkey maintained fixation during the stimulus (eye movements were $<0.5^{\circ}$ ) and indicated the direction of motion with an eye movement after the stimulus was extinguished.

2.2 Data Analysis. We analyzed 54 cells in three monkeys: $\mathrm{E}$ ( $n=$ $26), \mathrm{J}(n=9)$, and $\mathrm{W}(n=19)$. Not all cells were recorded under all experimental conditions, so the number of cells involved in each analysis will be stated in the text. Cells with mean responses that changed by more than $100 \%$ during recording sessions were not included. In all computations, the post-stimulus time histogram (PSTH) for a set of spike trains is the average number of action potentials as a function of time relative to stimulus onset and is computed at the same millisecond resolution as the original recordings-the data are not smoothed. The PSTHs shown in the figures, however, have been smoothed using an adaptive square window that is widened to include a criterion number of spikes. A onesided estimate of the power spectral density of the PSTH is computed using the standard fast Fourier transform (FFT) algorithm and overlapping data segments with windowing (Press et al. 1988). To avoid biasing our statistics with the initial transient response, we restrict our analysis, except where noted, to the $1600 \mathrm{msec}$ "sustained" portion of the spike trains that follows the $400 \mathrm{msec}$ "transient" period beginning at stimulus 
onset, $t=0$. Bursts of action potentials (consecutive spikes occurring with interspike intervals of 3 msec or less) can create excessive power at low frequencies for some neurons and therefore are replaced by single action potentials using the technique described by Bair et al. (1994).

This analysis focuses on properties of sets of spike trains that were recorded from single neurons using the same dynamic dot sequence. For illustration and as a control, sets of responses for randomly seeded stimuli, but with all other stimulus parameters held constant, are analyzed and referred to as "control" data. The control data should not show signs of stimulus-locked temporal modulation in the PSTH because responses to different stimuli are averaged together. However, the control data provide a null hypothesis that is better than a Poisson assumption because known deviations from Poisson temporal structure, e.g., refractory periods, bursts, and drifts in excitability, will be present.

A simple test for a violation of Poisson statistics was used to determine the presence of stimulus-locked modulation in a set of spike trains. The average firing rate was determined for the $1600 \mathrm{msec}$ sustained period and taken to be the mean rate of a homogeneous Poisson point process. If the observed firing rate in any segment of the sustained period was improbably high at the $10^{-6}$ significance level, the response was considered to have stimulus-locked modulation. The stringent significance level reflects the inadequacy of the Poisson process to account for the refractory period, burst firing, and nonstationarities that are frequently found in spike trains. Of the 54 cells, 49 were found to respond to a dynamic dot sequence with significant temporal modulation based on 30 trials at $i=0$. The test for significant modulation, when run on 54 sets of control data taken from the same number of cells in the same animals, yielded two false positives. Visual inspection of these false positives revealed that the spike rate changed slowly over the course of the trial and that this trend was independent of the particular dot pattern.

\section{Results}

A neuron presented with 90 different random dot stimuli at $c=0$ produced an ensemble of responses (Fig. 1, left) that, except for the initial transient, can be approximated by a point process with a time-invariant mean rate, such as a homogeneous Poisson process modified by a refractory period (Bair et al. 1994). The right side of Figure 1, showing 90 responses of the same cell to one particular $c=0$ random-dot stimulus, reveals that the neuron's firing pattern was often very tightly locked to the stimulus. Thus, much of the apparent randomness of the ensemble on the left was caused by the fact that a different random dot pattern was presented on each trial, although some periods of the response remain unstructured. The firing pattern on the right can be modeled to first order by a random process with a time varying mean rate, such as 
an inhomogeneous Poisson process. The time-varying modulation, estimated by the PSTH (bottom right), is characterized by narrow peaks, often produced by single action potentials occurring at precise instants across trials. A second neuron responded to a dynamic dot sequence at $c=0$ with a much higher firing rate but was still highly modulated (Fig. 2).

After characterizing the temporal modulation in the time and frequency domains, we will contrast the patterned responses in Figure 1 and Figure 2 with the case of $c=1$ stimulation, i.e., coherent motion, in which modulation is not present when an identical stimulus is repeated.

3.1 Precision and Reliability. For the 49 cells which had statistically significant stimulus-locked modulation (see Section 2.2), we quantified the temporal precision of the spike trains using the standard deviation (SD) in time of the onset of periods of elevated firing such as those indicated by peaks in the PSTH at the bottom right of Figure 1. This technique is similar to that used by Sestokas and Lehmkuhle (1986). The standard deviation measure will be referred to as temporal "jitter" and has a small value for a precise response.

A peak in the PSTH corresponding to a period of significantly elevated firing probability (see Section 2.2) was accepted as well enough isolated for analysis if an arbitrary point in time preceding the peak existed such that the mean time to the first spike in the response was greater than twice the standard deviation of the distribution of first spike times. For example, one statistically significant peak is marked by a thick line near $1740 \mathrm{msec}$ in the PSTH at the bottom right of Figure 1. Considering a period of $70 \mathrm{msec}$ surrounding that peak, we measure the time from the beginning of the period to the first spike on each trial. The distribution of first spike times is shown in Figure 3A. The SD, or jitter, is $3.3 \mathrm{msec}$ and yet no action potential occurs for at least $25 \mathrm{msec}$ prior to the response. The distribution achieved by this method is different from the shape of the peak in the PSTH, which includes all the spikes, not just the first one in a response. In addition, by considering only the first spike in an isolated response period on each trial, our measurement is less likely to be biased by a refractory period or the interspike interval statistics for the neuron. For a few neurons, such as the one in Figure 2, the significant peaks in the PSTH were not well enough isolated to perform the jitter analysis for individual action potentials. In those cases, we searched for the first occurrence of a pair of spikes with less than a specified interspike interval (6 msec in Fig. 2). The distribution of the occurrence of these pairs is shown in Figure 3B.

Reliability was measured as the probability that a response occurred during the periods described above. For the response period analyzed in Figure 3A, the cell responded (fired at least one action potential) on $24 \%$ of trials (see Fig. 1, right, for spike trains). The same cell had other, more reliable responses-the reliability for the peak near $1000 \mathrm{msec}$ was 

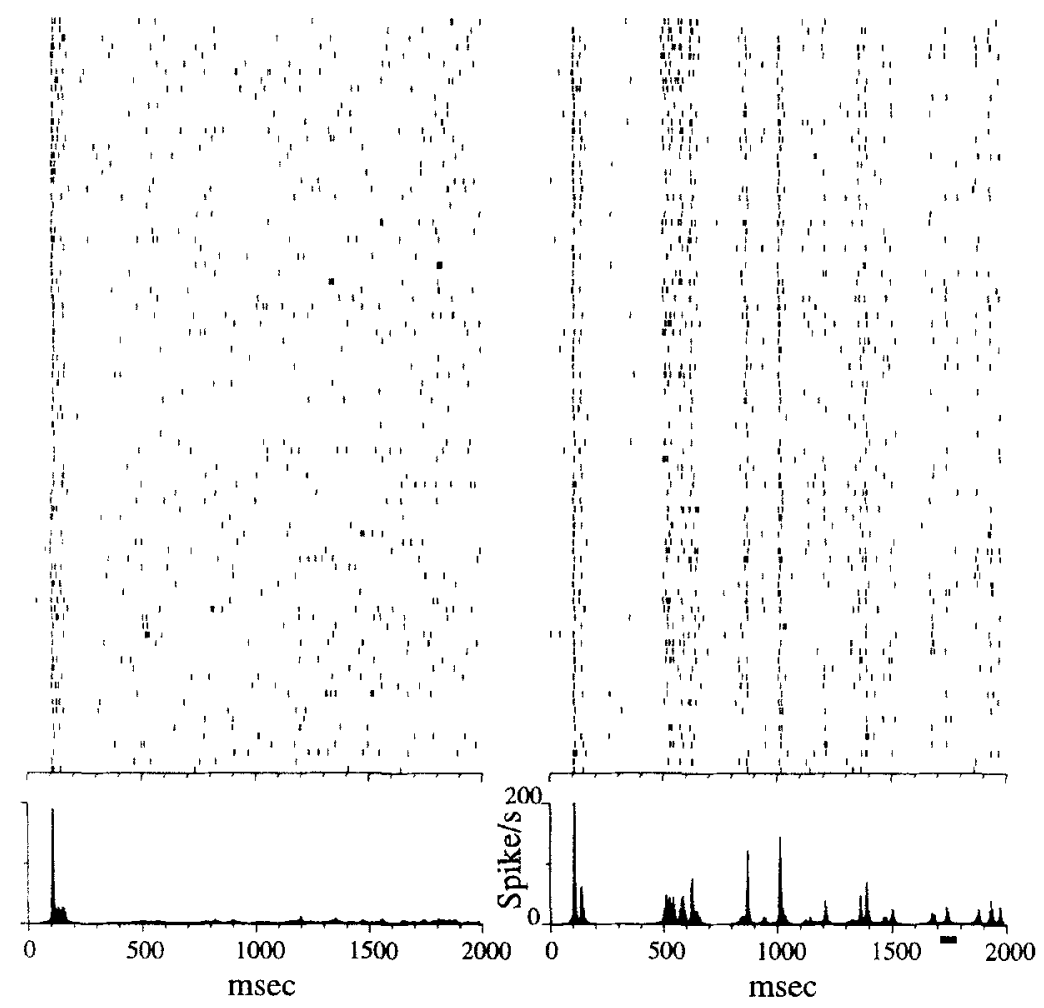

Figure 1: The neuronal response of one cell in area MT in a behaving macaque monkey to randomly seeded dynamic dot stimuli at $c=0$ presented for $2 \mathrm{sec}$ (left) appears to be well described by a point process with a mean rate of $3.4 \mathrm{~Hz}$ (excluding the initial transient). However, when a dynamic dot stimulus formed with a particular seed was repeated (but interleaved with different stimuli) many times, the reliability of the response became apparent (right). Viewing this figure from an acute angle reveals the precision of the pattern; for example, nearly all spikes in the final $400 \mathrm{msec}$ of the response cluster in six vertical streaks. Below each set of spike trains is a poststimulus time histogram (PSTH) computed from each set of 90 trials using an adaptive square window centered at each point and widened to capture 10 spikes. The set of data on the left is referred to as control data.

0.84. Within the response period marked on the PSTH for the neuron in Figure 2, two spikes with interspike interval $\leq 6 \mathrm{msec}$ occurred on $95 \%$ of the trials. The distributions shown in Figure $3 \mathrm{~A}$ and $\mathrm{B}$ correspond to the 


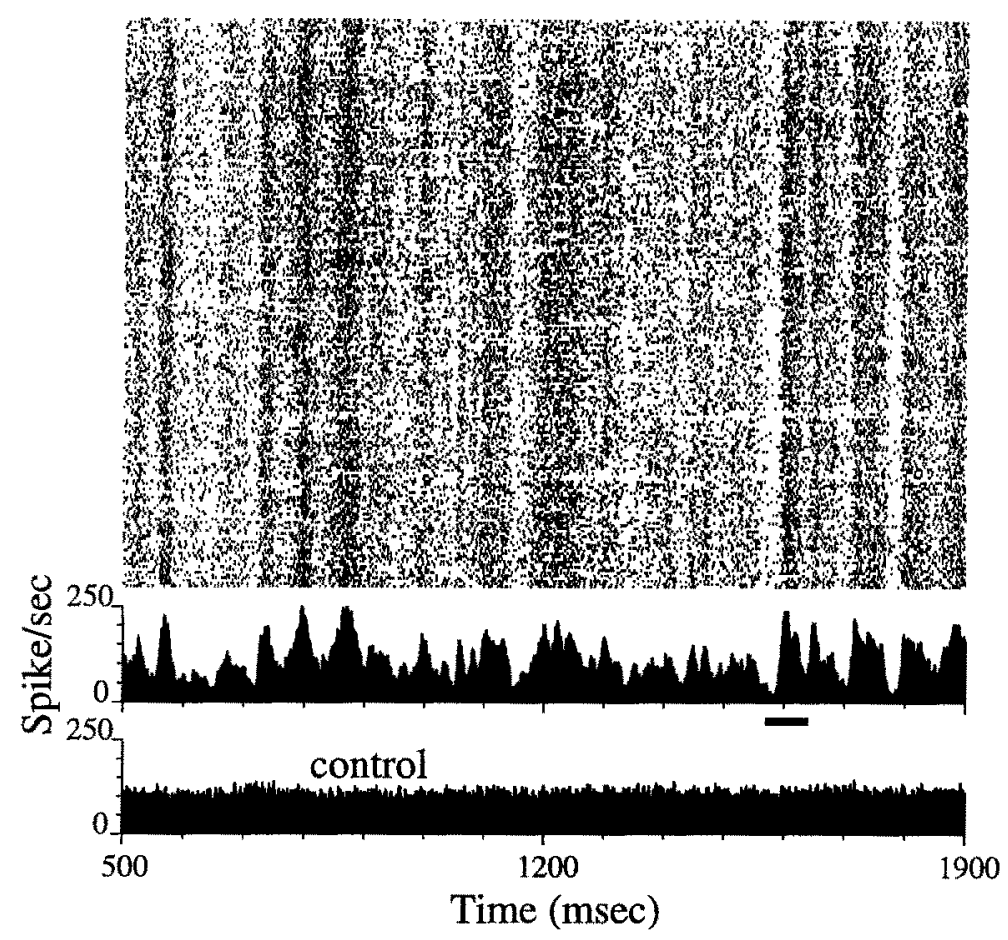

Figure 2: The neuronal response of another MT cell to $c=0$ dynamic dot stimulation on 206 trials using a particular stimulus pattern. This neuron (e093) produces a highly modulated response, like the neuron in Figure 1, but has a much higher firing rate $(113 \mathrm{~Hz}$, SD $15 \mathrm{~Hz}, 206$ trials). While the responses of most cells contained occasional clearly isolated epochs of elevated firing rate, this cell never dips down to its background firing rate $(2 \mathrm{~Hz})$. The temporal precision of the response peak indicated by the thick bar below the PSTH is shown in Figure 3, and the power spectra for both PSTHs are shown in Figure 4. The lower, flat PSTH corresponds to 210 trials of control data. The adaptive window used to smooth the PSTHs is widened to include 40 spikes.

most precise responses for the spike trains shown in Figure 1 (right) and Figure 2. For all 49 cells, the scatter plot in Figure 3C shows reliability versus jitter for the most precise response during the sustained period (filled circles) and for the initial transient, present in only 32 of 49 cells (crosses). For $80 \%$ of cells, the most precise response during the sustained period had jitter less than $10 \mathrm{msec}$ and in some cases the jitter was as 

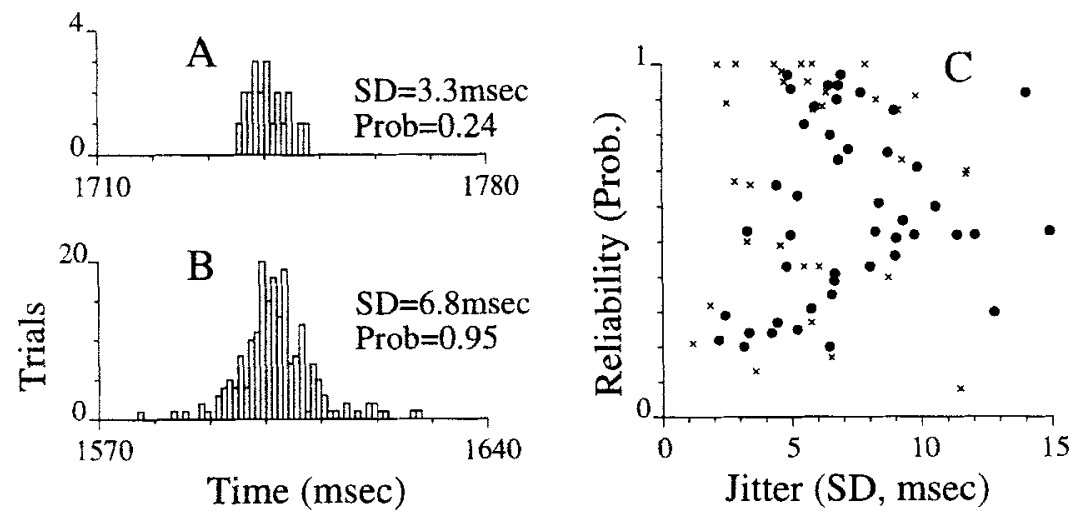

Figure 3: MT neurons are temporally precise on the order of milliseconds. We measured precision as the standard deviation (SD), or jitter, of the beginning or ending time of a period of elevated firing, e.g., the periods indicated by the thick lines below the PSTHs at $1750 \mathrm{msec}$ in Figure 1 and near $1600 \mathrm{msec}$ in Figure 2. (A) The distribution across trials of the occurrence time for the first action potential in the response during the period $1710-1780 \mathrm{msec}$ in Figure 1. (B) The distribution of the occurrence time of the first pair of action potentials fired within $6 \mathrm{msec}$ in the time period $1570-1640 \mathrm{msec}$ in Figure 2. (C) The jitter for the most precise response periods is plotted against the response reliability (probability) for 49 cells (solid circles). In $80^{\circ} \%$ of cells, the minimum temporal jitter during the sustained portion of the response was less than $10 \mathrm{msec}$, with the smallest values near $2-3 \mathrm{msec}$. For comparison, crosses indicate the jitter of initial transients (present in only 32 of 49 cells). At least 30 repeated trials were used for each cell. Four points exceeded the horizontal scale, with jitter values of $16,19,27$, and 69 msec.

small as $2-4 \mathrm{msec}$. The initial transients, when present, typically had less jitter than the most precise sustained period response.

3.2 Frequency Profile. The previous analysis focused only on the most precise period of the responses in the time domain, but we now examine the entire sustained period response in the frequency domain. Temporal frequency profiles of the responses of the MT cells were computed as the power spectra of the PSTHs for $c=0$ stimuli. Spectra are shown in Figure 4 for the PSTHs in Figure 1 (right) and Figure 2. The power spectra are consistent with the notion that the cells act as low pass filters for the white noise dot stimulus; however, it is important that these profiles are not mistaken for temporal frequency tuning curves (see 

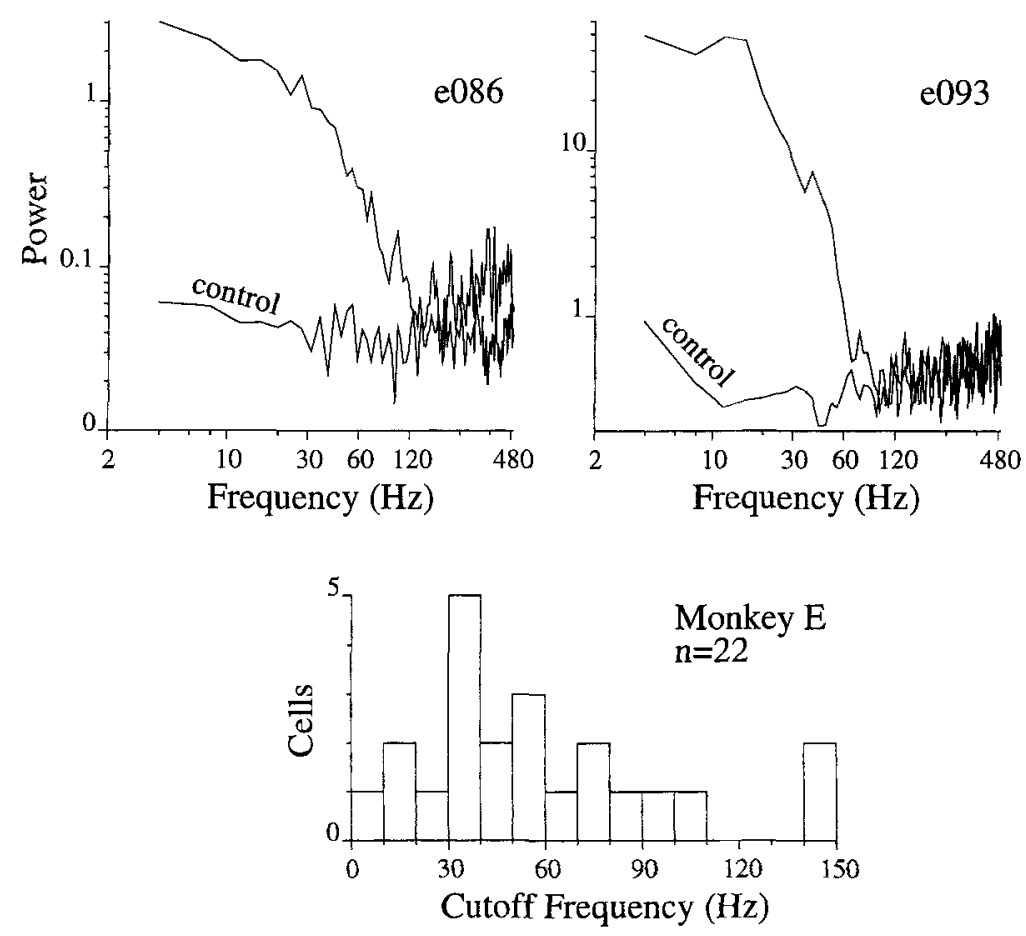

Figure 4: Temporal frequency cutoffs for area MT cells in response to dynamic random dots. The upper panels show the power spectra of the PSTHs for the neuronal responses shown in Figure 1 (right) and Figure 2. We defined the cutoff frequency for each cell as the lowest frequency at which the control spectrum intersects the response spectrum. The lower panel shows the distribution of frequency cutoffs for 22 cells from monkey E. (Monkey E was the only animal for which control data were available, see text.)

Discussion). There were no systematic peaks in the spectra at particular frequencies; for example, there was no stimulus refresh artifact as the dots were plotted asynchronously.

To compute an upper cutoff frequency for individual cells, we compared the power spectrum for a particular stimulus pattern to that for a PSTH computed from control data in which all trials resulted from different $c=0$ stimuli. The cutoff frequency was taken to be the lowest frequency at which the control power spectrum intersected the response power spectrum. The histogram of cutoff frequencies for 22 cells from monkey $E$ reveals a range of values from 0 to $150 \mathrm{~Hz}$ (mean \pm standard 
deviation $=58 \pm 38 \mathrm{~Hz}$, Fig. 4, bottom). Response data and control data from the same cell were available only for monkey $E$, but data recorded for individual coherent $(c=1)$ motion stimuli for $J$ and $W$ served as a control because, as reported in the next section, modulation was virtually absent for coherent motion. The distribution of cutoff frequencies for nine cells from monkey $\mathrm{J}$ had a mean $(46 \pm 10 \mathrm{~Hz})$ that was not significantly different from that for $E$ ( $t$ test, $p=0.19)$, while the mean $(23 \pm 12 \mathrm{~Hz})$ for six cells from $\mathrm{W}$ was significantly lower than that for both $\mathrm{E}$ and $\mathrm{J}(t$ test, $p<0.005$ ).

The distribution of cutoff frequencies shown in Figure 4 is consistent with our analysis of data from other experiments, using a different display, in which $73 \%$ of cells ( 22 of 30 ) in monkey J showed a peak in their power spectrum at $40 \mathrm{~Hz}$ when a coherent dot stimulus $(c=1)$ was presented in frames at $40 \mathrm{~Hz}$. In an experiment using moving bars, $24 \%$ of cells (12 of 49) in a fourth monkey $(R)$ showed peaks in their spectra at $60 \mathrm{~Hz}$ when the bar moved on a $60 \mathrm{~Hz}$ frame-refresh monitor.

Because the autocorrelation of a function is the Fourier transform of its power spectrum, these results may be interpreted in the time domain from the autocorrelation of the PSTH. The autocorrelations (computed after subtracting the means from the PSTHs) displayed a single peak before falling to zero with a width at half-height of $36 \pm 20$ msec (means for individual animals were: E $25 \pm 9.2$, J $33 \pm 16, W 50 \pm 24$ ). Note that both the autocorrelation function and the power spectrum are computed from the PSTH, and this is different from computing these functions for individual trials and averaging afterward (as done by Bair et al. 1994).

3.3 Response to Coherent Motion. The presence of temporal modulation depended on the motion coherence of the stimulus. While it was apparent for low coherence stimuli as shown in Figures 1 and 2, it was absent for highly coherent motion, i.e., $c=1$ (Fig. 5). We defined a measure $M$ of the overall modulation strength based on the power spectrum of the PSTH. $M$ was the integral of the power spectrum in the $4-30 \mathrm{~Hz}$ band divided by the mean spike rate across the PSTH. As shown in Figure 6 and by the range of cutoff frequencies in Figure 4, temporal modulation showed up as excessive contributions to this frequency band. In monkeys $J$ and $W$, repeated stimulation using a particular dot pattern was performed at higher coherence levels, $c=0.5$ and 1.0 , in addition to $c=0$. In both animals, the modulation strength $M$ was not significantly different at $c=0$ versus $c=0.5(p>0.20$, paired $t$ test). Yet, for both animals, $M$ was significantly less at $c=1$ compared to $c=0.5$ (statistical significance: $p<0.005$ for monkey $\mathrm{J}, p<0.05$ for monkey $W$ ). ${ }^{1}$

\footnotetext{
${ }^{1}$ Because $c=1.0$ stimulation was not used for all cells; these computations were based on nine cells in monkey $J$ and six cells in monkey $W$. In all computations, the average $M$ computed for control data from other cells within the same animal was subtracted.
} 


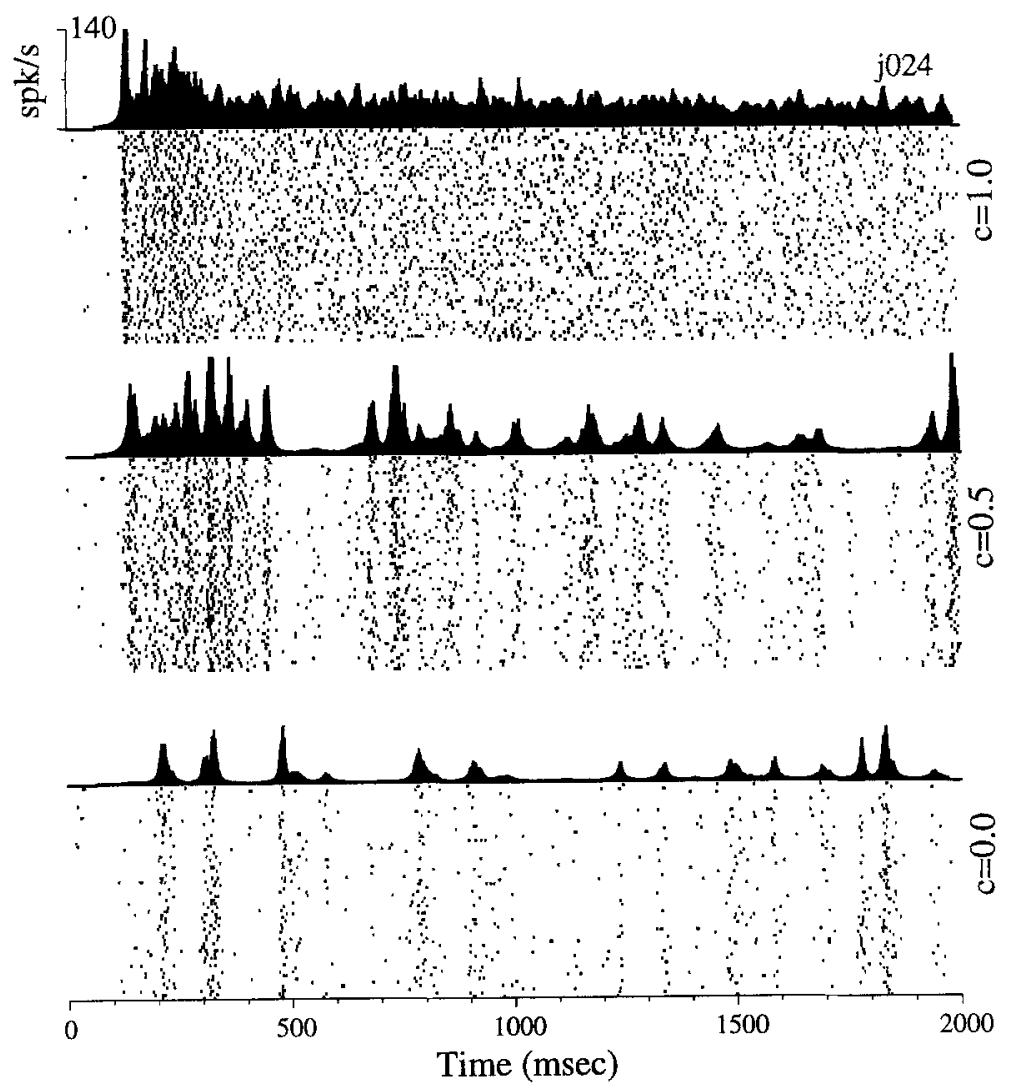

Figure 5: Temporal modulation disappears for highly coherent stimuli. The spike trains and PSTHs demonstrate that the stimulus-locked temporal modulation present for incoherent motion $(c=0)$ and for partially coherent motion ( $c=0.5$ ) was virtually absent during the sustained period of the response to coherent motion $(c=1)$. This suggests that temporal dynamics of a higher order than those found in rigid translation are necessary to induce a specific and unique time course in the spike discharge pattern.

We note that $M$ was not significantly correlated with spike rate ( $r=$ $0.09, p=0.50, M$ vs. $\log$ of spike rate) nor with the diameter of the stimulus aperture, which was optimized for the receptive field of each cell $(r=0.15, p=0.31)$. 


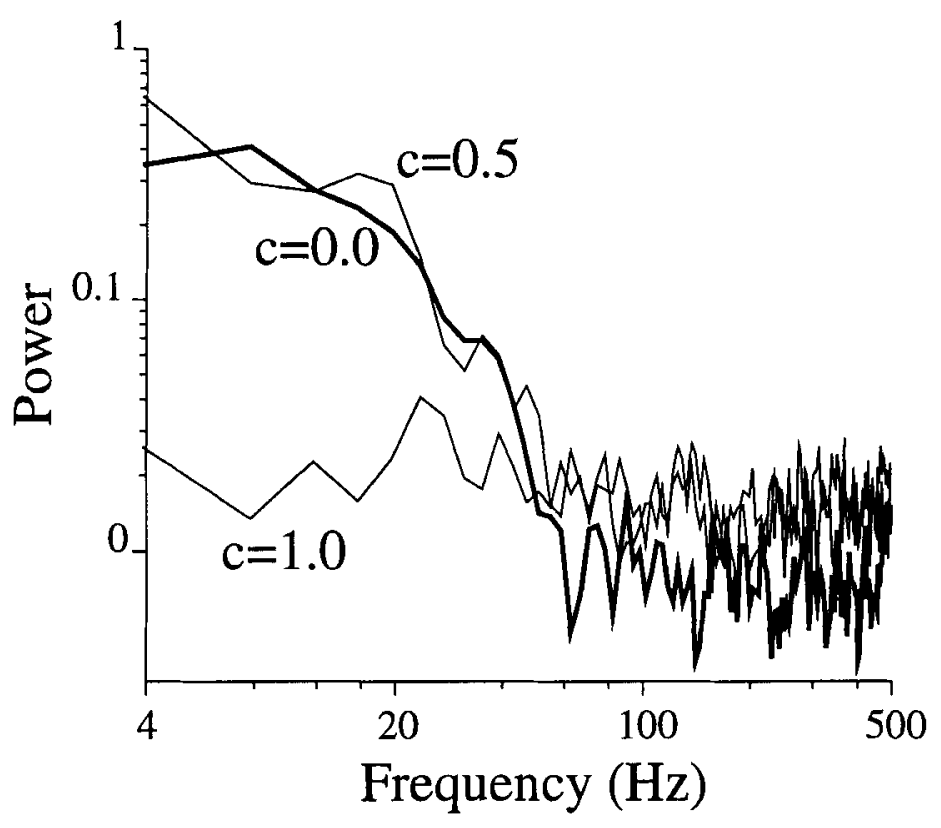

Figure 6: Power spectra of the PSTHs in Figure 5. The temporal modulation, reflected by excess power at frequencies below $60 \mathrm{~Hz}$, was similar for $c=0$ and $c=0.5$ but was absent for coherent motion. Curves for values between $c=0$ and $c=0.5$ (not shown) are similar to those shown for $c=0$ and $c=0.5$. Rarely were data recorded at values between $c=0.5$ and $c=1.0$, so intermediate curves cannot be shown. The PSTHs were expressed as instantaneous firing probabilities and the resulting power spectra were normalized by the mean firing rate. Our measure $M$ of temporal modulation was the integral of the scaled spectrum in the $4-30 \mathrm{~Hz}$ band. Fifty-nine spike trains were included at each $c$. The first $400 \mathrm{msec}$ of the PSTHs (shown in Fig. 5) was excluded to eliminate the initial transient response, which would otherwise contribute power in the $4-30 \mathrm{~Hz}$ band for all $c$.

\section{Discussion}

We have observed that a dynamic dot stimulus can produce periods of precise modulation in neurons in area MT. While it is common practice to seek the stimulus that causes the "largest activity" (Lettvin et al. 1959), we have sought those periods in which the stimulus caused the most precise activity in order to estimate the temporal precision with which the cortical network can trigger an action potential within a neuron. Using the standard deviation measure of temporal jitter, we found that $80 \%$ of 
cells were capable of responding with jitter less than $10 \mathrm{msec}$, and the most precise responses had jitter less than $2 \mathrm{msec}$. The reliability of the cell, its probability of contributing to a peak in the PSTH on a particular trial, varied widely from 0 to 1 (Fig. 3).

The output frequency profiles of the averaged responses of MT cells (Fig. 4) are all low-pass with a broad range of cutoffs, some above $100 \mathrm{~Hz}$, while the visual input provided by the incoherent dynamic dot stimulus has a flat temporal frequency spectrum. ${ }^{2}$ We emphasize that the frequency profiles of the responses reported here should not be interpreted as temporal frequency tuning curves. We do not know, for example, whether power near $50 \mathrm{~Hz}$ in the response is caused by $50 \mathrm{~Hz}$ components in the stimulus or arises from a computation such as squaring or rectifying a $25 \mathrm{~Hz}$ input component. ${ }^{3}$ However, it is not unreasonable to assume that area MT processes information that has frequency components in the $100 \mathrm{~s}$ of $\mathrm{Hz}$ since apparent motion can be perceived for temporal separations of less than $5 \mathrm{msec}$ (Baker and Braddick 1985). Even under the assumption that all inputs to area MT have relatively low cutoff frequencies, high-frequency signals may still be reconstructed from the spatial distributions of the inputs, in analogy to hyperacuity. But there is no lack of evidence that precise and high-frequency responses are carried by single neurons earlier on. Fast initial transients (having 50$100 \mathrm{~Hz}$ oscillations) are observed in magnocellularly derived responses in V1 (Maunsell and Gibson 1992) and, in the cat, retinal ganglion cells can lock their output to $100 \mathrm{~Hz}$ flicker (Eysel and Burandt 1984).

The stimulus-locked temporal modulation that accompanies incoherent and partially coherent motion is not present for coherent motion, even though the same dots are flashed at the same time and location from one presentation of the coherent stimulus to the next and in a manner such that only a single moving dot is likely to traverse a V1 subunit of an MT receptive field at any time (within $50 \mathrm{msec}$ or longer). In terms of the output, spatial inhomogeneities in the MT receptive field (so called "hot spots"), if they exist, are not apparent for this type of rigid pattern translation. The lack of hot spots is consistent with our models of MT receptive fields (see below). We do not believe that the lack of modulation for $c=1$ is the result of saturation of the neuron under study because narrow peaks exist in cross-correlograms between pairs of simultaneously recorded neurons at $c=1$ (Ehud Zohary, unpublished observations). Snowden et al. (1992), using a smaller, much denser stimulus ( $3^{\circ}$ aperture), estimated that $90 \%$ of MT cells did not modulate to a moving random dot stimulus; however, because the motion coherence of their stimulus is approximately equivalent to that of a $c=0.96$ stimulus here, their results are consistent with the lack of modulation

\footnotetext{
${ }^{2}$ In practice, the approximate $150 \mu \mathrm{sec}$ lifetime of the dots is so short that the departure from a flat temporal spectrum is not significant to the monkey visual system.

${ }^{3}$ Squaring a sine wave causes frequency doubling, and rectification introduces even higher harmonics.
} 
that we find at $c=1.0$. For coherent motion in the neuron's preferred direction, the neuronal response seems to lack precise temporal structure, and only the mean spike count is reproducible. In this case, the timing of individual action potentials may be governed by noise, but if more careful experimentation reveals that the spikes are precisely locked to internal fluctuations in the cortical network, we may realize, as Barlow (1972) observed for individual nerve cells, that "their apparently erratic behavior was caused by our ignorance, not the neuron's incompetence."

The data reported here have some analogy to that recorded in other studies that have been successful in estimating how much signal is carried in seemingly variable neuronal discharge. In the work of Bialek et al. (1991), a random motion stimulus also produced stimulus-locked temporal modulation with precision on the order of milliseconds (although their analysis was not limited to this time scale). In subsequent experiments in primary afferents of the cricket cercus and of the bullfrog sacculus, it was found that at least half of the entropy in the spike trains carried information regarding the stimulus (Rieke et al. 1993). Their analysis relied on estimates of the limit of timing precision of an action potential, which they found to be $0.4 \mathrm{msec}$ in the cricket and $2 \mathrm{msec}$ in the frog. It will be interesting to determine whether the precision of the responses reported here will yield similar results for visual cortical neurons and how coding efficiency will vary across different visual stimuli and visual areas. Of course, these computations require knowledge of the time-varying input signal, which is not available for the current data set.

In other work (Bair 1995) we examined how the temporal modulation reported here compares to the output of a motion energy model operating on the dynamic dot stimuli. Using a model that spatially integrates the output of opponent motion energy units (Adelson and Bergen 1985) across an area the size of an MT receptive field (5-10 diameter), it is possible to achieve modulation with precision and power spectra similar to that shown here in Figures 3 and 4 for incoherent, i.e., $c<1$, stimuli (Bair 1995). The model also accounts for the lack of modulation in response to $c=1$ stimuli. The lack of modulation in the model output is not the result of saturation but is a consequence of integration over an even and sufficiently dense (as specified by the sampling theorem) grid of motion energy units.

Although the statistics of a motion energy based model can be made to match those of the data reported here, it is important to consider that the model was constructed of noiseless linear filters and perfect multiplications and additions, with the only stochastic element being the generation of Poisson impulses from the analog output at the final stage of the model. Therefore, we believe it remains a challenge to theories of cortical processing (Stevens 1994) to explain how the observed low probabilities of synaptic transmission in cortical brain slices (Smetters and Nelson 1993; Thomson et al. 1993; Allen and Stevens 1994), combined with single synaptic contacts among pyramidal cells in mammalian cor- 
tex (Freund et al. 1985; Andersen 1990; Gulyas et al. 1993), can give rise to these highly reproducible spike patterns in cells roughly 7 to 8 synapses remote from the sensory periphery over a 2-hr-long experiment in a behaving animal (see also Lestienne and Strehler 1987; Abeles et al. 1993). Mainen and Sejnowski (1995) report that sustained current injection into cells in neocortical slice leads to a variable spike response, while repeated stimulation with a particular white noise current evokes a reliable spike pattern. Their findings in cortical cells suggest that the spike triggering mechanism itself is capable of accurately encoding temporally modulated input into spike trains, possibly providing the biophysical substrate of our results. It remains to be determined to what extent the rapid temporal modulation reported here carries any detailed information of behavioral significance.

\section{Acknowledgments}

We thank William T. Newsome for kindly providing data collected in his laboratory and for extensive critical discussion that has shaped the course of this analysis. Kenneth H. Britten, Michael N. Shadlen, Ehud Zohary, and J. Anthony Movshon have contributed greatly through critical discussion and by sharing data that they have collected. This work was funded by the Office of Naval Research and the Air Force Office of Scientific Research. W. B. was supported by the L. A. Hanson Foundation.

\section{References}

Abeles, M., Bergman, H., Margalit, E., and Vaadia, E. 1993. Spatiotemporal firing patterns in the frontal cortex of behaving monkeys. J. Neurophysiol. 70; $1629-1638$.

Adelson, E. H., and Bergen, J. R. 1985. Spatiotemporal energy models for the perception of motion. J. Opt. Soc. Am. A. 2, 284-299.

Adrian, E. 1928. The Basis of Sensation: The Action of Sense Organs. Christophers, London.

Allen, C., and Stevens, C. F. 1994. An evaluation of causes for unreliability of synaptic transmission. Proc. Natl. Acad. Sci. U.S.A. 91, 10380-10383.

Andersen, P. 1990. Synaptic integration in hippocampal CA1 pyramids. Prog Brain Res. 83, 215-222.

Bair, W. 1995. Analysis of temporal structure in spike trains of visual cortical area MT. Ph.D. Dissertation, Department of Computation and Neural Systems, California Institute of Technology, Pasadena, CA.

Bair, W., Koch, C., Nesome, W. T., and Britten, K. H. 1994. Power spectrum analysis of bursting cells in area MT in the behaving monkey. J. Neurosci. 14, 2870-2892.

Baker, C. L., and Braddick, O. J. 1985. Eccentricity-dependent scaling of the limits for short-range apparent motion perception. Vision Res. 25, 803-812. 
Barlow, H. B. 1972. Single units and perception: A neuron doctrine for perceptual psychology: Perception 1, 371-394.

Bialek, W., Rieke, F., de Ruyter van Steveninck, R. R., and Warland, D. 1991. Reading a neural code. Science 252, 1854-1857.

Britten, K. H., Shadlen, M. N., Newsome, W. T., and Movshon, J. A. 1992. The analysis of visual motion: A comparison of neuronal and psychophysical performance. I. Neurosci. 12, 4745-4765.

Britten, K. H., Shadlen, M. N., Newsome, W. T., and Movshon, J. A. 1993. Response of neurons in macaque MT to stochastic motion signals. Visual Neurosci. 10, 1157-1169.

Derrington, A. M., and Lennie, P. 1984. Spatial and temporal contrast sensitivities of neurones in lateral geniculate nucleus of macaque. J. Physiol. 357, 219-240.

Eysel, U. T., and Burandt, U. 1984. Fluorescent tube light evokes flicker responses in visual neurons. Vision Res. 24, 943-948.

Foster, K. H., Gaska. J. P., Nagler, M., and Pollen, D. A. 1985. Spatial and temporal frequency selectivity of neurones in visual cortical areas V1 and V2 of the macaque monkey. J. Physisl. 365, 331-363.

Freund, T. F., Martin, K. A. C., and Whitteridge, D. 1985. Innervation of cat visual area- 17 and area- 18 by physiologically identified $X$-type and Y-type thalamic afferents. 1. Arborization patterns and quantitative distribution of postsynaptic elements. I. Comp. Neurol. 242, 263-274.

Gulyas, B., Orban, G. A., Duysens, J., and Maes, H. 1987. The suppressive influence of moving textured backgrounds on responses of cat striate neurons to moving bars. J. Neurophysiol. 57, 1767-1791.

Gulyas, A. I., Miles, R., Sik, A., Toth, K., Tamamaki, N., and Freund, T. F. 1993. Hippocampal pyramidal cells excite inhibitory neurons through a single release site. Nature (London) 366, 683-687.

Hammond, P., and Mackay, D. M. 1977. Differential responsiveness of simple and complex cells in cat striate cortex to visual texture. Exp. Brnin Res. 30, 275-296.

Heggelund, P., and Albus, K. 1978. Response variability and orientation discrimination of single cells in striate cortex of cat. Exp. Brain Res. 32, 197-211.

Henry, G. H., Bishop, P. O., Tupper, R. M., and Dreher, B. 1973. Orientation specificity and response variability of cells in the striate cortex. Vision Res. 13, 1771-1779

Lee, B. B., Martin, P. R., and Valberg, A. 1989. Sensitivity of macaque retinal ganglion cells to chromatic and luminance flicker. J. Physiol. 414, 223-243.

Lestienne, R., and Strehler, B. L. 1987. Time structure and stimulus dependence of precisely replicating patterns present in monkey cortical neuronal spike trains. Broin Re's. 437, 214-238.

Lettvin, J. P., Maturana, H. R., McCulloch, W. S., and Pitts, W. H. 1959. What the frog's eye telis the frog's brain. Proc. Inst. Rat. Eng. 47, 1950-1961.

Levitt, J. B., Kiper, D. C., and Movshon, J. A. 1994. Receptive fields and functional architecture of macaque V2. J. Neturophiss. 71, 2517-2542.

Mainen, Z. F., and Sejnowski, T. J. 1995. Reliability of spike timing in neocortical neurons. Science 268, 1503-1506. 
Maunsell, J. H. R., and Gibson, J. R. 1992. Visual response latencies in striate cortex of the macaque monkey. J. Neurophysiol. 68, 1332-1344.

McLean, J., and Palmer, L. A. 1989. Contribution of linear spatiotemporal receptive field structure to velocity selectivity of simple cells in area 17 of cat. Vision Res. 29, 675-679.

Newsome, W. T., Britten, K. H., and Movshon, J. A. 1989. Neuronal correlates of a perceptual decision. Nature (London) 341, 52-54.

Press, H. P., Flannery, B. P., Teukolsky, S. A., and Vetterling, W. T. 1988. Numerical Recipes in $C$, the Art of Scientific Computing. Cambridge University Press, Cambridge.

Richmond, B. J., Optican, IL. M., Podell, M., and Spitzer, H. 1987. Temporal encoding of two-dimensional patterns by single units in primate inferior temporal cortex. I. Response characteristics. J. Neurophysiol. 57, 132-146.

Richmond, B. J., Optican, L. M., and Spitzer, H. 1990. Temporal encoding of two-dimensional patterns by single units in primate primary visual cortex. I. Stimulus-response relations. J. Neurophysiol. 64, 351-369.

Rieke, F., Warland, D., and Bialek, W. 1993. Coding efficiency and information rates in sensory neurons. Europhys. Lett. 22, 151-156.

Schiller, P. H., Finlay, B. L., and Volman, S. F. 1976. Short-term response variability of monkey striate neurons. Brain Res. 105, 347-349.

Sclar, G., Maunsell, J. H. R., and Lennie, P. 1990. Coding of image contrast in central visual pathways of the macaque monkey. Vistal Res. 30, 1-10.

Sestokas, A. K., and Lehmkuhle, S. 1986. Visual response latency of X- and Y-cells in the dorsal lateral geniculate nucleus of the cat. Vision Res. 26, 1041-1054.

Shadlen, M. N., and Newsome, W. T. 1994. Noise, neural codes and cortical organization. Curr. Opin. Neurobiol. 4, 569-579.

Smetters, D. K., and Nelson, S. B. 1993. Estimates of functional synaptic convergence in rat and cat visual cortical neurons. Soc. Neurosci. Abstr. 19, 628.

Snowden, R. J., Treue, S., and Anderson, R. A. 1992. The response of neurons in areas $\mathrm{V} 1$ and $\mathrm{MT}$ of the alert rhesus monkey to moving random dot patterns. Exp. Brain Res, 88, 389-400.

Softky, W. R., and Koch, C. 1993. The highly irregular firing of cortical cells inconsistent with temporal integration of random EPSPs. J. Neurosci. 13, 334-350.

Stevens, C. F. 1994. What form should a cortical theory take? In Large-Scale Nelironal Theories of the Brain, C. Koch and J. L. Davis, eds., pp. 239-255. MIT Press, Cambridge, MA.

Thomson, A. M., Deuchars, J., and West, D. C. 1993. Large, deep layer pyramidpyramid single axon EPSPs in slices of rat motor cortex display pairedpulse and frequency-dependent depression, mediated presynaptically and self-facilitation, mediated postsynaptically. J. Neurophysiol. 70, 2354-2369.

Tolhurst, D. J., Movshon, J. A., and Thompson, I. D. 1981. The dependence of response amplitude and variance of cat visual cortical neurones on stimulus contrast. Exp. Brain Res. 41, 414-419.

Tolhurst, D. J., Movshon, J. A., and Dean, A. F. 1983. The statistical reliability 
of signals in single neurons in cat and monkey visual cortex. Vision Res. 23, $775-785$.

Tomko, G. J., and Crapper, D. R. 1974. Neuronal variability: Non-stationary responses to identical visual stimuli. Brain Res. 79, 405-418.

Vogels, R., Spileers, W., and Orban, G. A. 1989. The response variability of striate cortical neurons in the behaving monkey. Exp. Brain Res. 77, 432-436.

Werner, $G_{1}$, and Mountcastle, V. B. 1963. The variability of central neural activity in a sensory sytem, and its implications for the central reflection of sensory events. J. Neurophysiol. 26, 958-977.

Received August 7, 1995; accepted February 2, 1996. 